Accepted: 2015.08.30

Published: 2015.12.03
Received: 2015.07 .01

\section{Genetic Polymorphisms Analysis of Pharmacogenomic VIP Variants in Miao Ethnic Group of Southwest China}

Authors' Contribution: Study Design A Data Collection B Statistical Analysis C Data Interpretation D Manuscript Preparation E Literature Search F Funds Collection G
AB 1,2,3,4 Tianbo Jin*

B 5 Ainiwaer Aikemu*

E 3,4 Mingxia Zhang

F 4 Tingting Geng

D 4 Tian Feng

$G$ 1,2 Longli Kang

A 6 Manlin Luo
1 Key Laboratory of Molecular Mechanism and Intervention Research for Plateau Diseases of Tibet Autonomous Region, School of Medicine, Xizang Minzu University, Xianyang, Shaanxi, P.R. China of Tibet Autonomous Region, School of Medicine, Xizang Minzu University, Xianyang, Shaanxi, P.R. China

3 School of Life Sciences, Northwest University, Xi'an, Shaanxi, P.R. China 4 National Engineering Research Center for Miniaturized Detection Systems, Xi'an, Shaanxi, P.R. China

5 Department of Drug Analysis, Faculty of Pharmacy, Xinjiang Medical University, Urumqi, Xinjiang, P.R. China

6 Department of Blood Transfusion, Yunnan Province Second People's Hospital, Kunming, Yunnan, P.R. China
2 Key Laboratory of High Altitude Environment and Genes Related to Diseases
Corresponding Author: Source of support:
* Tian bo Jin and Ainiwaer Aikemu are joint first authors Man lin Luo, e-mail: manlinluo@163.com

This work was supported by the Major Training Program of Xizang Minzu University (No. 13myZP06), Natural Science Foundation of Xizang (Tibet) Autonomous Region (20152R-13-11), Major science and technology research projects of Xizang (Tibet) Autonomous Region (2015), and the National Natural Science Foundations (No. 81560516)
Material/Methods:

Results:

Conclusions:

MeSH Keywords:

Full-text PDF:
Genetic polymorphisms have a potential clinical role in determining both inter-individual and inter-ethnic differences in drug efficacy, but we have not found any pharmacogenomics information regarding minorities, such as the Miao ethnic group. Our study aimed to screen numbers of the Miao ethnic group for genotype frequencies of VIP variants and to determine differences between the Miao and other human populations worldwide. In this study, we genotyped 66 Very Important Pharmacogene (VIP) variants selected from PharmGKB in 98 unrelated, healthy Miao individuals from the Guizhou province and compared our data with 12 other populations, including 11 populations from the HapMap data set and Xi'an Han Chinese.

Using the $\chi^{2}$ test, we found that the allele frequencies of the VDR rs1544410 and VKORC1 (rs9934438) variants in the Miao population are quite different from that in other ethnic groups. Furthermore, we found that genotype frequencies of rs1801133 (MTHFR) in the 13 selected populations are significantly different. Population structure and F-statistics (Fst) analysis show that the genetic background of the Miao is relatively close to that of Chinese in metropolitan Denver, CO, USA (CHD).

Our results help complete the information provided by the pharmacogenomics database of the Miao ethnic group and provide a theoretical basis for safer drug administration, which may be useful for diagnosing and treating diseases in this population.

\section{Ethnic Groups • Genetic Counseling • Genomic Structural Variation}

http://www.medscimonit.com/abstract/index/idArt/895191

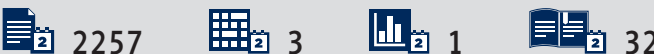




\section{Background}

The large variability among individuals in drug efficacy is a major challenge in current clinical practice, drug development, and drug regulation [1]. It has been suggested that genetic background may be responsible for the variation in response to therapy, and mounting evidence demonstrates that an individual's genetic makeup accounts for an estimated 20 95\% of variability in drug disposition and effects [2,3]. Pharmacogenetics and pharmacogenomics elucidated the inherited nature of individual variation in drug response, with the goal of optimizing efficacy and safety through better understanding of human genetic variability and its influence on drug response, leading to personalized medicine $[4,5]$.

The Pharmacogenetics and Pharmacogenomics Knowledge Base (PharmGKB: http://www.pharmgkb.org) is a publicly available Web-based knowledge base created to aid researchers in understanding how genetic variation among individuals contributes to differences in reactions to drugs [6]. This information is presented in the form of Very Important Pharmacogene (VIP) summaries, pathway diagrams, and curated literature [7]. The PharmGKB currently contains information for more than 3000 drugs, 3000 diseases, and 26000 genes with genotyped variants [8]. In total, it consists of $126 \mathrm{VIP}$ variants that occur in 44 different genes and variously code for cytochrome P450 oxidases, drug targets, drug receptors, and drug transporters. The relationship between these VIP variants and their effect on drug-related toxicity as well as therapeutic benefit have been studied extensively [9].

Pharmacogenomic research in ethnic populations has great significance for the achievement of personalized drug treatment and development of new drugs. However, we have not found any pharmacogenomics information regarding minority groups, such as the Miao ethnic groups in southwest China. The Miao is an ethnic group mainly distributed in the southwest of China; they mostly live in Guizhou, Yunnan, and Sichuan provinces. It is one of China's largest ethnic groups, with a long history, distinct culture, and fine traditions. According to a 2000 census, the Miao have an approximate population of 9.6 million.

In the present study, we aimed to identify the allele frequencies of VIP variants in the Miao and to determine the difference in allele frequencies between the Miao and 12 other populations. Our goals were to identify differences and determine their extent and provide a theoretical basis for safer drug administration and better therapeutic treatment in the Miao population. The results of our study will extend our understanding of ethnic diversity and pharmacogenomics, and help clinicians triage patients for better individualized treatments.

\section{Material and Methods}

\section{Study participants}

We randomly recruited 98 unrelated, healthy Miao subjects from Guizhou province of China. The subjects selected were judged to be of good health and had exclusively Miao ancestry for at least the last 3 generations. We selected 96 unrelated Chinese Han individuals from Lantian county in Xi'an, Shaanxi province as one of our control groups. All subjects were healthy in terms of their medical history and physical examination. An explanation about the purpose and experimental procedures of the study were given to all individuals. Written informed consent was obtained from all subjects prior to sample donation, and the study protocol was performed in accordance with the Declaration of Helsinki and approved by the Clinical Research Ethics of Northwest University for Approval of Research Involving Human Subjects.

\section{Variant selection and genotyping}

We selected genetic variants from published polymorphisms associated with VIP variants from the Pharm GKB database, and excluded loci that could not be designed. We successfully genotyped 66 VIP variants selected from PharmGKB in 194 participants (98 Miao subjects and 96 Chinese Han controls). Genomic DNA was isolated from whole blood using the GoldMag-Mini Whole Blood Genomic DNA Purification Kit (GoldMag Ltd. Xi'an, China) according to the manufacturer's protocol. DNA concentration was measured by NanoDrop 2000C (Thermo Scientific, Waltham, Massachusetts, USA). We used the Sequenom MassARRAY Assay Design 3.0 software (San Diego, CA, USA) to design Multiplexed SNP MassEXTEND assays [10]. Single-nucleotide polymorphism (SNP) genotyping used the standard protocol recommended by the manufacturer with a Sequenom MassARRAY RS1000 (San Diego, California, USA). Sequenom Typer 4.0 Software (San Diego, California, USA) was used to perform data management and analyze the SNP genotyping data, as described in a previous report [11].

\section{HapMap genotype data}

The genotype data of the 11 populations were downloaded from the International HapMap Project web site (HapMap_release127) at http://hapmap.ncbi.nlm.nih.gov. The 11 populations are as follows: (1) African ancestry in Southwest USA (ASW); (2) Utah, USA residents with Northern and Western European ancestry from the CEPH collection (CEU); (3) Han Chinese in Beijing, China (CHB); (4) Chinese in metropolitan Denver, CO, USA (CHD); (5) Gujarati Indians in Houston, Texas, USA (GIH); (6) Japanese in Tokyo, Japan (JPT); (7) Luhya in Webuye, Kenya (LWK); (8) Mexican ancestry in Los Angeles, California, USA 
(MEX); (9) Maasai in Kinyawa, Kenya (MKK); (10) Toscani in Italy (TSI); and (11) Yoruba in Ibadan, Nigeria (YRI).

\section{Data analysis}

We used Microsoft Excel (Redmond, WA, USA) and SPSS 17.0 statistical packages (SPSS, Chicago, IL, USA) to perform statistical calculations. The validity of the frequency of each VIP variant in the Miao and Chinese Han data was tested by assessing the departure from HWE using an exact test. We calculated and compared the genotype frequencies of the variants in the Miao data with those in the 11 populations separately using the $\chi^{2}$ test [12]. All $p$ values obtained in this study were 2-sided, and Bonferroni adjustment for multiple tests was applied to the level of significance, which was set at $p<0.05 /\left(66^{*} 12\right)$ [13]. Structure (version 2.3.4) software [14] was used to analysis the genetic structure of the 13 populations. We used Arlequin (version 3.1) software to calculate the value of Fst to infer the pairwise distance between populations [15].

\section{Results}

We successfully sequenced 66 VIP pharmacogenomic variant genotypes from 98 Miao individuals. The basic information about the selected VIP loci in Miao is listed in Table 1, including gene name and category, chromosome number and position, amino acid translation, and their allele frequencies in Miao.

We used the $\chi^{2}$ test with the Bonferroni correction for multiple hypotheses and multiple comparisons, and we found $5,7,12$, $13,14,15,15,16,16,19,19$, and 25 different loci in the frequency distributions when the Miao population was compared to the Xi'an Han, CHD, MEX, ASW, JPT, CHB, GIH, MKK, TSI, CEU, LWK, and YRI populations, respectively $\left(p \leq 6.3 \times 10^{-5}\right)$. These VIP variants are mainly distributed in 23 genes; they mainly involve the cytochrome P450 superfamily, nuclear receptor family, G-protein-coupled receptor family, alcohol dehydrogenase family, adrenergic receptors family, ATP-binding cassette (ABC) transporters superfamily, and eag family. Genotype frequencies of MTHFR, VDR, and VKORC1 in the Miao differed widely from those in the other 12 populations. We found that the rs1801133 was the most significantly different locus between the Miao ethnic group and the other populations (Table 2). Additionally, Rs698, Rs1805124, and Rs 1801131 were found to show a significant difference in the 11 HapMap populations.

Pairwise Fst values were calculated for all population comparisons across loci. As shown in Table 3, we found that pairwise Fst values for comparisons of the Miao population with the other 12 populations ranged from 0.01904 to 0.26192 . Fst statistics [16] are measures of population differentiation. It is directly related to the variance in allele frequency among populations and to the degree of resemblance among individuals within populations. If Fst is small, it means that the allele frequencies within each population are similar; if it is large, it means that the allele frequencies are different. The value of Fst for the Miao and CHD populations was the smallest. We therefore conclude that the allele frequencies of the Miao and CHD are similar. We speculate that the genetic backgrounds of the Miao and CHD populations are similar.

We used a model-based clustering approach, as implemented in Structure, to infer population structure among the 13 populations. Different values ranging from 2 to 7 were assumed for $\mathrm{K}$ in Structure calculations. $\mathrm{K}=3$ was selected, based on the estimated Ln Prob of Data and other recommendations of the Structure software manual. As shown in Figure 1, when the $\mathrm{K}$ value was equal to 3 , individuals were independently assigned to 3 affinity groups (subpopulations 1: Miao, Xi'an Han, CHB, CHD, JPT; subpopulations 2: ASW, LWK, MKK, YRI; subpopulations 3: CEU, GIH, MEX, TSI) using the relative majority of likelihood to assign individuals to subpopulations. We tested additional values of $\mathrm{K}$ and obtained results suggesting that the genetic backgrounds of the Miao and CHD populations are similar.

\section{Discussion}

Individuals' differences in drug reactions can directly influence the efficacy and safety of the drug, and has become a worldwide problem in the treatment of some major diseases. However, it is almost impossible to predict whether a drug will be beneficial, lack efficacy, or cause serious adverse effects [17]. Because genetic variations play an important role in determining the metabolism of and reactions to some specific drugs in individual patients, in this study we genotyped the variants related to drug response (pharmacogenomics) in the Miao ethnic group and compared the genotype frequencies with those in 12 other populations. The $\chi^{2}$ test results show that the allele frequencies of the VDR rs1544410 and VKORC1 (rs9934438) variants in the Miao population are quite different from that in other ethnic groups. We found that genotype frequencies of rs1801133 (MTHFR) in the 13 selected populations are significantly different. Using Fst calculations and analysis of population structure, we also found that the genetic backgrounds of the Miao and CHD population are similar.

Methylenetetrahydrofolate reductase (MTHFR), located on chromosome 1 at $1 p 36.3$, is an important enzyme involved in the folate metabolic pathway. Rs1801133 (677 C>T) is a significant variant of the MTHFR gene. In our present study, rs1801133 was found to be a significant variant that existed in the 13 selected populations. It has been widely reported that the polymorphism of rs1801133 is associated with many diseases, 
Table 1. Basic information about the selected variants and allele frequencies in the Miao ethnicity.

\begin{tabular}{|c|c|c|c|c|c|c|c|c|c|c|}
\hline \multirow{2}{*}{ SNP ID } & \multirow{2}{*}{ Genes } & \multicolumn{2}{|l|}{ Category } & \multirow{2}{*}{$\begin{array}{l}\text { Amino acid } \\
\text { translation }\end{array}$} & \multirow{2}{*}{$\begin{array}{l}\text { Chromo- } \\
\text { some }\end{array}$} & \multirow{2}{*}{ Position } & \multicolumn{2}{|c|}{ Allele } & \multicolumn{2}{|c|}{ Allele frequencies } \\
\hline & & Family & Phase & & & & $\mathbf{A}$ & B & A (\%) & B (\%) \\
\hline rs1801131 & MTHFR & $\begin{array}{l}\text { Methylenetetrahydrofolate } \\
\text { reductase }\end{array}$ & Phase I & Glu429Ala & 1 & 11854476 & c & A & 1 & 0 \\
\hline rs1801133 & MTHFR & $\begin{array}{l}\text { Methylenetetrahydrofolate } \\
\text { reductase }\end{array}$ & Phase I & Ala222Val & 1 & 11856378 & $T$ & c & 0.72 & 0.28 \\
\hline rs20417 & PTGS2 & Nuclear receptor & Others & - & 1 & 186650320 & G & c & 1 & 0 \\
\hline rs 689466 & PTGS2 & Nuclear receptor & Others & - & 1 & 186650750 & A & G & 0.49 & 0.51 \\
\hline rs3918290 & DPYD & - & Phase I & - & 1 & 97915614 & G & I & 1 & 0 \\
\hline rs 6025 & F5 & - & Others & Arg534Gln & 1 & 169519049 & c & A & 1 & 0 \\
\hline rs 890293 & CYP2J2 & Cytochrome P450 & Phase I & - & 1 & 60392494 & G & $T$ & 0.34 & 0.66 \\
\hline rs4148323 & UGT1A10 & UDP-glucuronosyltransferase & Phase li & Gly71Arg & 2 & 234669144 & A & G & 0.23 & 0.77 \\
\hline rs1065776 & $\mathrm{P} 2 \mathrm{RY} 1$ & G-protein coupled receptor & Others & Ala19Ala & 3 & 152553628 & $\mathrm{~T}$ & C & 0.96 & 0.04 \\
\hline rs2046934 & P2RY12 & G-protein coupled receptor & Others & - & 3 & 151057642 & T & c & 0.19 & 0.81 \\
\hline rs3814055 & NR112 & Nuclear receptor & Others & - & 3 & 119500034 & C & $\mathrm{T}$ & 0.91 & 0.09 \\
\hline rs 1805124 & SCN5A & Sodium channel gene & Others & Pro1090Leu & 3 & 38645420 & G & A & 0.85 & 0.15 \\
\hline rs6791924 & SCN5A & Sodium channel gene & Others & Arg34Cys & 3 & 38674699 & G & I & 1 & 0 \\
\hline rs7626962 & SCN5A & Sodium channel gene & Others & Ser1103Tyr & 3 & 38620907 & G & I & 1 & 0 \\
\hline rs975833 & $\mathrm{ADH} 1 \mathrm{~A}$ & Alcohol dehydrogenase & Phase I & - & 4 & 100201739 & G & C & 0.75 & 0.25 \\
\hline rs1229984 & $\mathrm{ADH} 1 \mathrm{~B}$ & Alcohol dehydrogenase & Phase I & His48Arg & 4 & 100239319 & G & A & 0.68 & 0.32 \\
\hline rs2066702 & $\mathrm{ADH} 1 \mathrm{~B}$ & Alcohol dehydrogenase & Phase I & Arg370Cys & 4 & 100229017 & c & $\mathrm{T}$ & 1 & 0 \\
\hline rs698 & $\mathrm{ADH} 1 \mathrm{C}$ & Alcohol dehydrogenase & Phase I & Ile350Val & 4 & 100260789 & A & G & 0.95 & 0.05 \\
\hline rs1042713 & ADRB2 & Adrenergic receptors & Others & Ala222Val & 5 & 148206440 & G & A & 0.48 & 0.52 \\
\hline rs1042714 & ADRB2 & Adrenergic receptors & Others & - & 5 & 148206473 & G & C & 0.98 & 0.02 \\
\hline rs1800888 & ADRB2 & Adrenergic receptors & Others & Thr164lle & 5 & 148206885 & C & $\mathrm{T}$ & 1 & 0 \\
\hline rs 17238540 & HMGCR & - & Phase I & - & 5 & 74619742 & $\mathrm{~T}$ & I & 1 & 0 \\
\hline rs 17244841 & HMGCR & - & Phase I & - & 5 & 74607099 & A & I & 0.99 & 0.01 \\
\hline rs3846662 & HMGCR & - & Phase I & - & 5 & 74615328 & $\mathrm{~T}$ & c & 0.57 & 0.43 \\
\hline rs 1142345 & TPMT & Methyltransferase superfamily & Phase li & Tyr240Cys & 6 & 18130918 & G & A & 0.99 & 0.01 \\
\hline rs1045642 & $\mathrm{ABCB} 1$ & $A B C$ transporters & Others & Ile1145Ile & 7 & 87138645 & $\mathrm{~T}$ & c & 0.65 & 0.35 \\
\hline rs1128503 & ABCB1 & $A B C$ transporters & Others & Gly412Gly & 7 & 87179601 & $\mathrm{~T}$ & C & 0.33 & 0.67 \\
\hline rs2066853 & AHR & AHR & Others & Arg554Lys & 7 & 17379110 & G & A & 0.52 & 0.48 \\
\hline rs12720441 & $\mathrm{KCNH} 2$ & Eag & Others & Arg444Trp & 7 & 150647304 & c & I & 1 & 0 \\
\hline rs36210421 & $\mathrm{KCNH} 2$ & Eag & Others & Arg707Leu & 7 & 150644428 & G & $\mathrm{T}$ & 1 & 0 \\
\hline rs3807375 & $\mathrm{KCNH} 2$ & Eag & Others & - & 7 & 150667210 & A & G & 0.78 & 0.22 \\
\hline rs3815459 & $\mathrm{KCNH} 2$ & Eag & Others & - & 7 & 150644394 & A & G & 0.73 & 0.27 \\
\hline rs 2740574 & CYP3A4 & Cytochrome P450 & Phase I & - & 7 & 99382096 & A & G & 0.99 & 0.01 \\
\hline rs12721634 & CYP3A4 & Cytochrome P450 & Phase I & Leu15Pro & 7 & 99381661 & $\mathrm{~T}$ & I & 1 & 0 \\
\hline rs4986909 & CYP3A4 & Cytochrome P450 & Phase I & Pro416Leu & 7 & 99359670 & C & I & 0.74 & 0.26 \\
\hline rs4986910 & CYP3A4 & Cytochrome P450 & Phase I & Met445Thr & 7 & 99358524 & $\mathrm{~T}$ & I & 1 & 0 \\
\hline rs10264272 & CYP3A5 & Cytochrome P450 & Phase I & Lys208Lys & 7 & 99262835 & c & I & 1 & 0 \\
\hline rs1801252 & ADRB1 & Adrenergic receptors & Others & Ser49Gly & 10 & 115804036 & G & A & 0.66 & 0.34 \\
\hline rs1799853 & CYP2C9 & Cytochrome P450 & Phase I & Arg144Cys & 10 & 96702047 & c & $\mathrm{T}$ & 1 & 0 \\
\hline
\end{tabular}


Table 1 continued. Basic information about the selected variants and allele frequencies in the Miao ethnicity.

\begin{tabular}{|c|c|c|c|c|c|c|c|c|c|c|}
\hline \multirow{2}{*}{ SNP ID } & \multirow{2}{*}{ Genes } & \multicolumn{2}{|l|}{ Category } & \multirow{2}{*}{$\begin{array}{l}\text { Amino acid } \\
\text { translation }\end{array}$} & \multirow{2}{*}{$\begin{array}{c}\text { Chromo- } \\
\text { some }\end{array}$} & \multirow{2}{*}{ Position } & \multicolumn{2}{|c|}{ Allele } & \multicolumn{2}{|c|}{ Allele frequencies } \\
\hline & & Family & Phase & & & & A & B & A (\%) & B (\%) \\
\hline rs4244285 & CYP2C19 & Cytochrome P450 & Phase I & Pro227Pro & 10 & 96541616 & G & A & 0.32 & 0.68 \\
\hline rs4986893 & CYP2C19 & Cytochrome P450 & Phase I & Trp212null & 10 & 96540410 & G & I & 0.015 & 0.985 \\
\hline rs1138272 & GSTP1 & Glutathione S-transferase & Phase li & Ala114Val & 11 & 67353579 & $\mathrm{~T}$ & c & 1 & 0 \\
\hline rs1695 & GSTP1 & Glutathione S-transferase & Phase li & Ile105Val & 11 & 67352689 & A & G & 0.889 & 0.121 \\
\hline rs1800497 & DRD2 & G-protein-coupled receptor & Others & Glu713Lys & 11 & 113270828 & $\mathrm{~T}$ & c & 0.59 & 0.41 \\
\hline rs6277 & DRD2 & G-rotein-coupled receptor & Others & Pro290Pro & 11 & 113283459 & G & A & 0.985 & 0.015 \\
\hline rs5219 & KCNJ11 & $\begin{array}{l}\text { Inward-rectifier potassium } \\
\text { channel family }\end{array}$ & Others & Lys23Glu & 11 & 17409572 & c & $\mathrm{T}$ & 0.939 & 0.061 \\
\hline rs11568820 & VDR & Nuclear receptor & Others & - & 12 & 48302545 & G & $A$ & 0.54 & 0.46 \\
\hline rs1540339 & VDR & Nuclear receptor & Others & - & 12 & 48257326 & G & A & 0.78 & 0.22 \\
\hline rs 1544410 & VDR & Nuclear receptor & Others & - & 12 & 48239835 & G & $A$ & 0.99 & 0.01 \\
\hline rs2239185 & VDR & Nuclear receptor & Others & - & 12 & 48244559 & $\mathrm{~T}$ & C & 0.78 & 0.22 \\
\hline rs9934438 & VKORC1 & VKORC1 & Phase I & - & 16 & 31104878 & G & A & 0.87 & 0.13 \\
\hline rs1801030 & SULT1A1 & Sulfotransferase & Phase li & Val223Met & 16 & 28617485 & A & I & 1 & 0 \\
\hline rs3760091 & SULT1A1 & Sulfotransferase & Phase li & - & 16 & 28620800 & C & G & 0.74 & 0.26 \\
\hline rs1801272 & CYP2A6 & Cytochrome P450 & Phase I & Leu160His & 19 & 41354533 & $\mathrm{~T}$ & I & 1 & 0 \\
\hline rs28399433 & CYP2A6 & Cytochrome P450 & Phase I & - & 19 & 41356379 & G & $\mathrm{T}$ & 0.23 & 0.77 \\
\hline rs28399444 & CYP2A6 & Cytochrome P450 & Phase I & Glu197Arg & 19 & 41354190 & A & I & 1 & 0 \\
\hline rs28399454 & CYP2A6 & Cytochrome P450 & Phase I & Val365Met & 19 & 41351267 & G & I & 1 & 0 \\
\hline rs28399499 & CYP2B6 & Cytochrome P450 & Phase I & Ile328Thr & 19 & 41518221 & $\mathrm{~T}$ & I & 1 & 0 \\
\hline rs3211371 & CYP2B6 & Cytochrome P450 & Phase I & Arg487Cys & 19 & 41522715 & c & $\mathrm{T}$ & 0.50 & 0.50 \\
\hline rs1051266 & SLC19A1 & Solute carrier & Others & His27Arg & 21 & 46957794 & G & A & 0.57 & 0.43 \\
\hline rs4680 & СОMT & COMT & Phase li & Val158Met & 22 & 19951271 & $A$ & G & 0.79 & 0.21 \\
\hline rs16947 & CYP2D6 & Cytochrome P450 & Phase I & - & 22 & 42523943 & G & A & 0.05 & 0.95 \\
\hline rs28371706 & CYP2D6 & Cytochrome P450 & Phase I & Thr107lle & 22 & 42525772 & $C$ & $\mathrm{~T}$ & 0.995 & 0.005 \\
\hline rs28371725 & CYP2D6 & Cytochrome P450 & Phase I & - & 22 & 42523805 & G & A & 0.05 & 0.95 \\
\hline rs5030656 & CYP2D6 & Cytochrome P450 & Phase I & - & 22 & 42524175 & AAG & I & 0.50 & 0.50 \\
\hline rs61736512 & CYP2D6 & Cytochrome P450 & Phase I & Val136Met & 22 & 42525134 & C & I & 1 & 0 \\
\hline
\end{tabular}

such as breast cancer [18], colorectal cancer [19], and bladder cancer [20]. A previous meta-analysis demonstrated that the $677 \mathrm{C}$ allele was significantly associated with breast cancer risk $(\mathrm{OR}=0.942,95 \% \mathrm{Cl}=0.898$ to 0.988$)$ when compared with the $677 \mathrm{~T}$ allele in the additive model [18]. In our study, the $C$ allele frequency in Miao was somewhat high (28\%) in our present study, suggesting that Miao have an intermediate susceptibility to breast cancer. Sohn et al. [21] demonstrated that the MTHFR 677T mutation decreased chemosensitivity of breast cancer cells to methotrexate (MTX), a common cancer chemotherapeutic agent. Cáliz et al. [22] also reported that the C677T polymorphism (rs1801133) was associated with increased MTX toxicity [odds ratio (OR) 1.42, 95\% confidence interval $(\mathrm{Cl}) 1.01-1.98, \mathrm{p}=0.0428$ ] in a Spanish rheumatoid arthritis population. These findings suggest that the MTHFR C677T polymorphism may be a useful pharmacogenetic determinant for providing rational and effectively tailored therapy for the Miao ethnic group.

Vitamin D receptor (VDR) gene maps to chromosome 12q13.11, whose function has been widely reported. It is an important regulator of the vitamin $D$ pathway and a number of common single-nucleotide polymorphisms (SNP) have been identified in this gene [23]. Clinical evidence suggests that the VDR genotype could modify the efficacy of anti-osteoporotic treatments such as etidronate and alendronate in postmenopausal women [24]. Other studies have demonstrated that the SNP rs1544410 in VDR might modulate the risk of breast, skin, and 
Table 2. Significant variants in Miao compared to the twelve populations determined by Chi-square test.

\begin{tabular}{|c|c|c|c|c|c|c|c|c|c|c|c|c|c|}
\hline \multirow{2}{*}{ SNP } & \multirow{2}{*}{ Genes } & \multicolumn{12}{|c|}{ Chi-square test p-value (after Bonferroni correction) } \\
\hline & & Xi'an Han & ASW & CEU & CHB & CHD & GIH & JPT & MEX & MKK & TSI & YRI & LWK \\
\hline rs1042713 & ADRB2 & - & $1.08 \mathrm{E}-05$ & - & 3.37E-06 & $5.00 \mathrm{E}-07$ & - & - & - & $1.35 \mathrm{E}-06$ & - & 4.63E-05 & 4.83E-05 \\
\hline rs1042714 & ADRB2 & - & - & $4.62 \mathrm{E}-18$ & $7.97 \mathrm{E}-29$ & - & - & $2.67 \mathrm{E}-28$ & - & - & - & $1.92 \mathrm{E}-29$ & - \\
\hline rs1045642 & $\mathrm{ABCB} 1$ & - & - & $1.02 \mathrm{E}-10$ & - & - & $2.39 \mathrm{E}-05$ & - & - & $8.43 \mathrm{E}-06$ & - & $1.21 \mathrm{E}-07$ & - \\
\hline rs1051266 & SLC19A1 & - & - & $3.05 \mathrm{E}-08$ & - & - & - & - & - & $9.94 \mathrm{E}-12$ & - & $2.11 \mathrm{E}-06$ & $4.12 \mathrm{E}-08$ \\
\hline rs1128503 & $\mathrm{ABCB} 1$ & - & $6.40 \mathrm{E}-14$ & $5.21 \mathrm{E}-07$ & - & - & - & - & - & $3.60 \mathrm{E}-25$ & $8.25 \mathrm{E}-06$ & $1.01 \mathrm{E}-24$ & $4.72 E-23$ \\
\hline rs1142345 & TPMT & - & 1.17E-32 & - & - & - & - & - & - & - & - & - & $8.35 \mathrm{E}-39$ \\
\hline rs11568820 & VDR & - & - & - & - & - & - & - & $1.34 \mathrm{E}-07$ & $9.98 \mathrm{E}-07$ & $1.01 \mathrm{E}-07$ & $1.52 \mathrm{E}-22$ & $9.39 \mathrm{E}-11$ \\
\hline rs1229984 & $\mathrm{ADH} 1 \mathrm{~B}$ & - & - & - & $1.35 \mathrm{E}-11$ & - & - & $2.86 \mathrm{E}-10$ & - & - & - & - & - \\
\hline rs1540339 & VDR & - & $1.15 \mathrm{E}-14$ & $3.35 \mathrm{E}-13$ & - & - & $4.27 \mathrm{E}-13$ & - & $9.34 \mathrm{E}-09$ & $1.64 \mathrm{E}-27$ & $5.39 \mathrm{E}-13$ & $3.91 \mathrm{E}-23$ & $1.05 \mathrm{E}-24$ \\
\hline rs 1544410 & VDR & $3.80 \mathrm{E}-41$ & $9.22 \mathrm{E}-32$ & $1.44 \mathrm{E}-27$ & - & - & $1.81 \mathrm{E}-29$ & $4.34 \mathrm{E}-39$ & $3.38 \mathrm{E}-29$ & $2.03 E-35$ & $6.31 \mathrm{E}-28$ & $2.01 \mathrm{E}-38$ & $9.08 \mathrm{E}-36$ \\
\hline rs1695 & GSTP1 & - & $8.63 \mathrm{E}-09$ & - & - & - & $2.39 \mathrm{E}-06$ & - & $4.02 \mathrm{E}-10$ & $6.13 \mathrm{E}-08$ & $1.65 \mathrm{E}-05$ & $5.05 \mathrm{E}-09$ & $1.46 \mathrm{E}-14$ \\
\hline rs1800497 & DRD2 & - & - & - & - & - & $4.40 \mathrm{E}-10$ & - & - & $6.38 \mathrm{E}-07$ & $2.20 \mathrm{E}-13$ & - & $1.32 \mathrm{E}-05$ \\
\hline rs1801131 & MTHFR & - & $1.03 \mathrm{E}-15$ & $3.89 \mathrm{E}-14$ & $4.97 \mathrm{E}-18$ & $6.97 \mathrm{E}-18$ & 1.10E-09 & $5.32 \mathrm{E}-20$ & $2.44 \mathrm{E}-14$ & $1.50 \mathrm{E}-19$ & $1.10 \mathrm{E}-12$ & $1.35 \mathrm{E}-27$ & $2.35 \mathrm{E}-20$ \\
\hline rs 1801133 & MTHFR & $2.75 \mathrm{E}-07$ & $5.58 \mathrm{E}-20$ & 9.97E-14 & $2.44 \mathrm{E}-05$ & $7.81 \mathrm{E}-11$ & 4.04E-20 & $2.99 \mathrm{E}-10$ & $5.34 \mathrm{E}-06$ & $2.44 \mathrm{E}-33$ & $5.93 \mathrm{E}-06$ & $9.64 \mathrm{E}-29$ & $5.70 \mathrm{E}-26$ \\
\hline rs1805124 & SCN5A & - & $1.03 \mathrm{E}-17$ & $8.80 \mathrm{E}-28$ & $6.45 \mathrm{E}-29$ & $2.36 \mathrm{E}-30$ & $9.16 \mathrm{E}-26$ & $3.79 \mathrm{E}-28$ & $3.71 \mathrm{E}-21$ & $3.48 \mathrm{E}-22$ & $4.53 \mathrm{E}-23$ & $2.71 \mathrm{E}-24$ & $2.47 \mathrm{E}-21$ \\
\hline rs 20417 & PTGS2 & - & - & $6.78 \mathrm{E}-32$ & - & - & - & - & - & - & - & $2.03 E-26$ & - \\
\hline rs2046934 & P2RY12 & - & - & $6.28 \mathrm{E}-17$ & $1.85 \mathrm{E}-18$ & - & - & $6.41 \mathrm{E}-19$ & - & - & - & $1.95 \mathrm{E}-20$ & - \\
\hline rs2066702 & $\mathrm{ADH} 1 \mathrm{~B}$ & - & $2.52 \mathrm{E}-30$ & - & - & - & - & - & - & $1.26 \mathrm{E}-52$ & - & $2.09 \mathrm{E}-40$ & $4.88 \mathrm{E}-40$ \\
\hline rs2066853 & AHR & - & - & $6.29 \mathrm{E}-08$ & - & - & $6.25 \mathrm{E}-13$ & - & $1.60 \mathrm{E}-08$ & - & 2.07E-14 & - & - \\
\hline rs2239185 & VDR & - & - & - & $8.08 \mathrm{E}-11$ & - & - & $8.76 \mathrm{E}-06$ & - & - & - & - & - \\
\hline rs28399454 & CYP2A6 & - & $1.62 \mathrm{E}-33$ & - & - & - & - & - & - & - & - & 4.13E-46 & $6.73 \mathrm{E}-41$ \\
\hline rs 28399499 & CYP2B6 & - & - & - & - & - & - & - & - & - & - & $7.82 \mathrm{E}-45$ & $1.50 \mathrm{E}-41$ \\
\hline rs3760091 & SULT1A1 & $5.37 \mathrm{E}-05$ & - & - & - & - & - & - & - & - & - & - & - \\
\hline rs3807375 & $\mathrm{KCNH} 2$ & - & - & $3.73 \mathrm{E}-16$ & - & - & $1.30 \mathrm{E}-12$ & - & - & - & $2.73 \mathrm{E}-14$ & - & - \\
\hline rs3814055 & NR112 & - & - & $3.96 \mathrm{E}-08$ & - & - & $7.55 \mathrm{E}-12$ & - & - & - & 5.27E-10 & $1.15 \mathrm{E}-05$ & $1.18 \mathrm{E}-05$ \\
\hline rs3815459 & $\mathrm{KCNH} 2$ & - & - & - & - & - & - & - & - & - & - & $1.15 \mathrm{E}-08$ & - \\
\hline rs3846662 & HMGCR & - & $1.11 \mathrm{E}-06$ & - & - & - & - & - & - & $1.12 \mathrm{E}-09$ & - & 4.95E-18 & $1.36 \mathrm{E}-16$ \\
\hline rs4148323 & UGT2A & - & - & - & $1.54 \mathrm{E}-19$ & $3.11 \mathrm{E}-20$ & $1.09 \mathrm{E}-31$ & $4.60 \mathrm{E}-23$ & $4.52 \mathrm{E}-23$ & - & - & $1.58 \mathrm{E}-28$ & - \\
\hline rs4244285 & CYP2C19 & - & - & $9.13 \mathrm{E}-07$ & $5.07 \mathrm{E}-10$ & - & - & $1.17 \mathrm{E}-08$ & - & - & - & $8.56 \mathrm{E}-17$ & - \\
\hline rs4680 & COMT & $9.20 \mathrm{E}-21$ & - & 8.99E-07 & - & - & $3.32 \mathrm{E}-06$ & - & - & - & 2.48E-07 & - & - \\
\hline rs4986909 & CYP3A4 & - & - & - & $9.66 \mathrm{E}-39$ & - & - & $3.55 \mathrm{E}-39$ & $2.29 \mathrm{E}-30$ & - & - & $2.20 \mathrm{E}-34$ & - \\
\hline rs5219 & KCNJ11 & $2.06 \mathrm{E}-05$ & - & - & - & - & - & - & - & - & - & - & - \\
\hline rs6277 & DRD2 & - & - & $4.12 \mathrm{E}-21$ & $6.85 \mathrm{E}-30$ & - & - & - & - & - & - & - & - \\
\hline rs689466 & PTGS2 & - & $1.12 \mathrm{E}-09$ & - & - & - & $2.92 \mathrm{E}-10$ & - & - & $1.22 \mathrm{E}-26$ & $6.81 \mathrm{E}-09$ & $4.29 \mathrm{E}-16$ & $1.69 \mathrm{E}-19$ \\
\hline rs698 & $\mathrm{ADH} 1 \mathrm{C}$ & - & $2.70 \mathrm{E}-23$ & $1.14 \mathrm{E}-21$ & $5.18 \mathrm{E}-34$ & $1.07 \mathrm{E}-33$ & $1.93 \mathrm{E}-28$ & $1.37 \mathrm{E}-33$ & $5.20 \mathrm{E}-23$ & $6.93 \mathrm{E}-38$ & $2.40 \mathrm{E}-25$ & $1.32 \mathrm{E}-39$ & $1.45 \mathrm{E}-31$ \\
\hline rs975833 & ADH1A & - & - & - & $2.11 \mathrm{E}-15$ & - & - & $1.00 \mathrm{E}-13$ & - & - & - & - & - \\
\hline rs9934438 & VKORC1 & - & - & $1.17 \mathrm{E}-07$ & $9.85 \mathrm{E}-34$ & $6.97 \mathrm{E}-33$ & - & $2.70 \mathrm{E}-31$ & $1.81 \mathrm{E}-08$ & - & $2.42 \mathrm{E}-11$ & - & - \\
\hline
\end{tabular}

prostate cancers, as well as other forms $[25,26]$. One study reported that GA and AA genotypes of rs 1544410 were associated with decreased cutaneous malignant melanoma (CMM) risk (odds ratio $=0.78$ and 0.75 , respectively) compared with the GG genotype [26]. We found that the GG genotype frequency of rs1544410 in the Miao is very high, suggesting that the Miao should consider more aggressive screening for CMM.

The VKORC1 (vitamin K epoxide reductase complex, subunit 1) gene encodes the VKORC1 (vitamin K epoxide reductase) 
Table 3. Pairwise Fst values between populations.

\begin{tabular}{|c|c|c|c|c|c|c|c|c|c|c|c|c|c|}
\hline Population & Miao & $\begin{array}{c}\text { Xi'an } \\
\text { Han }\end{array}$ & ASW & CEU & CHB & CHD & GIH & JPT & LWK & MEX & MKK & TST & YRI \\
\hline Miao & 0.00000 & & & & & & & & & & & & \\
\hline \multicolumn{14}{|c|}{ Xi'an Han 0.033820 .00000} \\
\hline ASW & 0.20416 & 0.20827 & 0.00000 & & & & & & & & & & \\
\hline CEU & 0.1826 & 0.15671 & 0.13819 & 0.00000 & & & & & & & & & \\
\hline CHB & 0.02257 & 0.00211 & 0.20498 & 0.15471 & 0.00000 & & & & & & & & \\
\hline CHD & 0.01904 & 0.00747 & 0.19789 & 0.14808 & -0.0012 & 0.00000 & & & & & & & \\
\hline GIH & 0.18045 & 0.17073 & 0.08734 & 0.02836 & 0.15889 & 0.15163 & 0.00000 & & & & & & \\
\hline JPT & 0.02566 & 0.01533 & 0.18516 & 0.14938 & 0.00547 & 0.00582 & 0.15292 & 0.00000 & & & & & \\
\hline LWK & 0.25869 & 0.27541 & 0.01665 & 0.20017 & 0.26908 & 0.26468 & 0.14679 & 0.24248 & 0.00000 & & & & \\
\hline MEX & 0.13783 & 0.09328 & 0.11843 & 0.02856 & 0.10109 & 0.09437 & 0.05388 & 0.10439 & 0.1902 & 0.00000 & & & \\
\hline MKK & 0.22133 & 0.23686 & 0.01934 & 0.14697 & 0.22869 & 0.22481 & 0.10441 & 0.19944 & 0.0145 & 0.1537 & 0.00000 & & \\
\hline TSI & 0.16704 & 0.13282 & 0.13316 & 0.0034 & 0.12909 & 0.1282 & 0.03023 & 0.12535 & 0.19757 & 0.02613 & 0.14577 & 0.00000 & \\
\hline YRI & 0.26192 & 0.28092 & 0.01823 & 0.21784 & 0.27486 & 0.27251 & 0.15499 & 0.24575 & 0.00361 & 0.20902 & 0.01936 & 0.21334 & 0.00000 \\
\hline
\end{tabular}

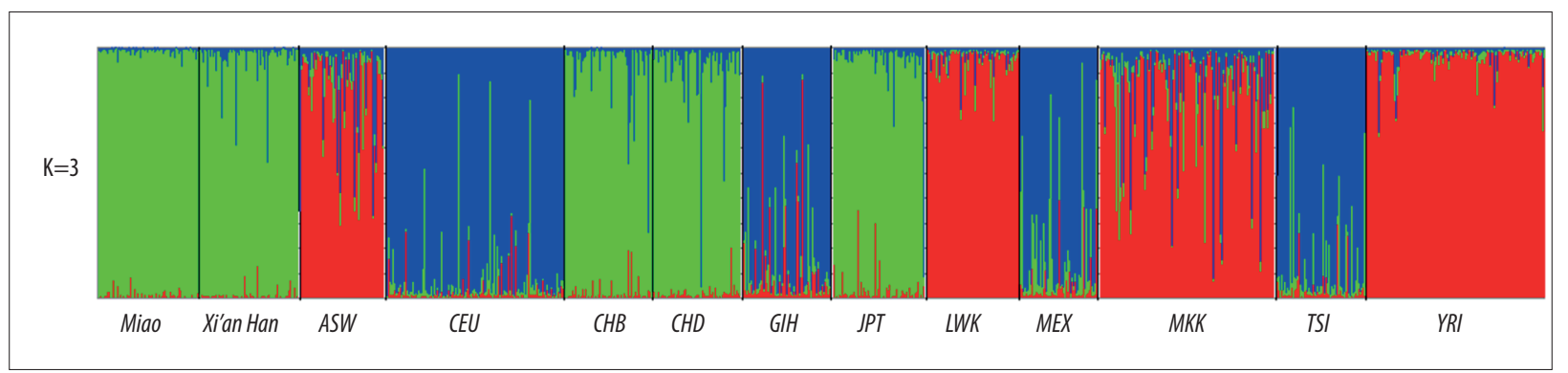

Figure 1. Bayesian clustering of genotypic samples from 13 populations. Each vertical bar denotes an individual and colors denote inferred clusters. Best model at $\mathrm{K}=3$, where the proportion of each ancestral component in a single individual is represented by a vertical bar divided into 3 colors.

protein, which is considered a candidate gene for the variability in warfarin response, mainly including 3 common polymorphisms [27]. The C6484T (rs9934438), or 1173C>T (rs9934438), is a SNP in the first intron of VKORC1, which was the first SNP associated with the low-dose warfarin phenotype [28]. A previous study demonstrated that patients with the 1173T (rs9934438) allele require a lower warfarin dose (mean dose 24-26 mg/week) compared with $35 \mathrm{mg} /$ week for the wild-type carriers [29]. In our study, the frequency of carriers of the allele T of rs9934438 is lower in the Miao population, suggesting that patients in this population will require a lower dose of warfarin.

Our study also demonstrated the correlation between the ethnic groups by Fst calculations and population structure analysis. The Structure plot (Figure 1) showed that the 13 ethnic groups were independently assigned into 3 affinity groups, suggesting they have a homogeneous genetic background. Genetic homogeneity among some populations separated by large geographic distances has been observed in migratory insects [30,31]. Our results are consistent with those findings, which could be explained by the migration theory described by Curry et al. [32].

Despite the current study possessing enough power, some limitations should be considered. First, the sample size of our study was relatively small, which may limit the statistical power. Second, the SNPs tested in our study were not large enough. Therefore, the association between these polymorphisms requires further investigation in a large sample before definitive conclusions can be drawn. 


\section{Conclusions}

Our results provide the first pharmacogenomics information in the Miao population and illustrate the difference in selected genes between Miao and 12 other populations around the world. These results could be used to create individualized

\section{References:}

1. Ma Q, Lu AY: Pharmacogenetics, pharmacogenomics, and individualized medicine. Pharmacol Rev, 2011; 63(2): 437-59

2. Marc J, Prezelj J, Komel R, Kocijancic A: VDR genotype and response to etidronate therapy in late postmenopausal women. Osteoporosis international: a journal established as result of cooperation between the European Foundation for Osteoporosis and the National Osteoporosis Foundation of the USA, 1999; 10(4): 303-6

3. Kalow W, Tang B, Endrenyi L: Hypothesis: comparisons of inter-and intra-individual variations can substitute for twin studies in drug research. Pharmacogenetics, 1998; 8(4): 283-89

4. Evans WE, McLeod HL: Pharmacogenomics - drug disposition, drug targets, and side effects. N Engl J Med, 2003; 348(6): 538-49

5. Crews KR, Hicks JK, Pui $\mathrm{CH}$ et al: Pharmacogenomics and individualized medicine: translating science into practice. Clin Pharmacol Ther, 2012; 92(4): 467-75

6. Whirl-Carrillo M, McDonagh E, Hebert J et al: Pharmacogenomics knowledge for personalized medicine. Clin Pharmacol Ther, 2012; 92(4): 414-17

7. Sangkuhl K, Berlin DS, Altman RB, Klein TE: PharmGKB: understanding the effects of individual genetic variants. Drug Metab Rev, 2008; 40(4): 539-51

8. Evans WE, Relling MV: Pharmacogenomics: translating functional genomics into rational therapeutics. Science, 1999; 286(5439): 487-91

9. Gan GG, Phipps ME, Lee MM et al: Contribution of VKORC1 and CYP2C9 polymorphisms in the interethnic variability of warfarin dose in Malaysian populations. Ann Hematol, 2011; 90(6): 635-41

10. Gabriel S, Ziaugra L, Tabbaa D: SNP genotyping using the Sequenom MassARRAY iPLEX platform. Curr Protoc Hum Genet, 2009; Chapter 2 :Unit 2.12

11. Thomas RK, Baker AC, DeBiasi RM et al: High-throughput oncogene mutation profiling in human cancer. Nat Genet, 2007; 39(3): 347-51

12. Adamec C: [Example of the use of the nonparametric test. Test $X 2$ for comparison of 2 independent examples.] Cesk Zdrav, 1964; 12: 613-19 [in Czech]

13. Song $M-K$, Lin F-C, Ward SE, Fine JP: Composite variables: when and how. Nurs Res, 2013; 62(1): 45-49

14. Falush D, Stephens M, Pritchard JK: Inference of population structure using multilocus genotype data: linked loci and correlated allele frequencies. Genetics, 2003; 164(4): 1567-87

15. Wright S: The genetical structure of populations. Annals of Eugenics, 1949 15(1): 323-54

16. Weir BS, Cockerham CC: Estimating F-statistics for the analysis of population structure. Evolution, 1984; 1358-70

17. Roden DM, Altman RB, Benowitz NL et al: Pharmacogenomics: challenges and opportunities. Ann Intern Med, 2006; 145(10): 749-57 treatment strategies, including appropriate drugs and dosage selections for the Miao ethnic group.

\section{Competing interests}

The authors declare that they have no competing interests.

18. Li K, Li W, Dong X: Association of $677 \mathrm{C}>\mathrm{T}$ ( $\mathrm{rs} 1801133)$ and $1298 \mathrm{~A}>\mathrm{C}$ (rs1801131) polymorphisms in the MTHFR gene and breast cancer susceptibility: a meta-analysis based on 57 individual studies. PloS One, 2014; 9(6): e71290

19. Teng Z, Wang L, Cai S et al: The $677 \mathrm{C}>\mathrm{T}$ (rs1801133) polymorphism in the MTHFR gene contributes to colorectal cancer risk: a meta-analysis based on 71 research studies. PloS One, 2013; 8(2): e55332

20. Xu W, Zhang H, Wang F, Wang H: Quantitative assessment of the association between MHTFR C677T (rs1801133, Ala222Val) polymorphism and susceptibility to bladder cancer. Diagn Pathol, 2013; 8: 95

21. Sohn K-J, Croxford R, Yates Z et al: Effect of the methylenetetrahydrofolate reductase C677T polymorphism on chemosensitivity of colon and breast cancer cells to 5-fluorouracil and methotrexate. J Natl Cancer Inst, 2004; 96(2): 134-44

22. Cáliz R, Del Amo J, Balsa A et al: The C677T polymorphism in the MTHFR gene is associated with the toxicity of methotrexate in a Spanish rheumatoid arthritis population. Scand J Rheumatol, 2012; 41(1): 10-14

23. Tworoger SS, Gate MA, Lee I-M et al: Polymorphisms in the vitamin D receptor and risk of ovarian cancer in four studies. Cancer Res, 2009; 69(5): 1885-91

24. Otrock ZK, Mahfouz RA, Charafeddine KM et al: Vitamin D receptor genotypes and response to zoledronic acid therapy in thalassemia-induced osteoporosis. Ann Hematol, 2008; 87(11): 947-48

25. Raimondi S, Johanson H, Maisonneuve P, Gandini S: Review and meta-analysis on vitamin $\mathrm{D}$ receptor polymorphisms and cancer risk. Carcinogenesis, 2009; 30(7): 1170-80

26. Gandini S, Raimondi S, Gnagnarella P et al: Vitamin D and skin cancer: a meta-analysis. Eur J Cancer, 2009; 45(4): 634-41

27. Rost $\mathrm{S}$, Fregin A, Ivaskevicius V et al: Mutations in VKORC1 cause warfarin resistance and multiple coagulation factor deficiency type 2 . Nature, 2004; 427(6974): 537-41

28. D'Andrea G, D'Ambrosio RL, Di Perna P et al: A polymorphism in the VKORC1 gene is associated with an interindividual variability in the dose-anticoagulant effect of warfarin. Blood, 2005; 105(2): 645-49

29. Wadelius M, Chen L, Downes K et al: Common VKORC1 and GGCX polymorphisms associated with warfarin dose. Pharmacogenomics J, 2005; 5(4): 262-70

30. Scott KD, Lawrence N, Lange CL et al: Assessing moth migration and population structuring in Helicoverpa armigera (Lepidoptera: Noctuidae) at the regional scale: example from the Darling Downs, Australia. Journal of Economic Entomology, 2005; 98(6): 2210-19

31. Llewellyn $\mathrm{K}$, Loxdale $\mathrm{H}$, Harrington R et al: Migration and genetic structure of the grain aphid (Sitobion avenae) in Britain related to climate and clonal fluctuation as revealed using microsatellites. Mol Ecol, 2003; 12(1): 21-34

32. Curry A: Coming to America. Nature, 2012; 485(7396): 30-32 